\title{
Chapter
}

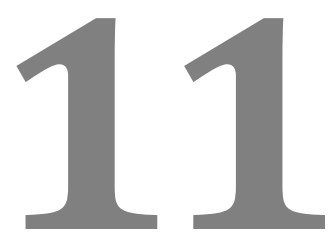

\section{EQUIBIOTICS: A NEW TYPE OF PHYTO-DRUG EQUILIBRATING LOCAL MICROBIOTA}

Xavier Lozoya, ${ }^{*}$ Maricela Gascón-Muro, Juan Agüero, and Erika Rivera-Arce

Phytomedicamenta Research Centre, México City, México 


\section{Contents}

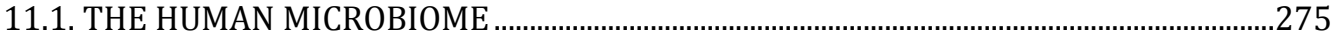

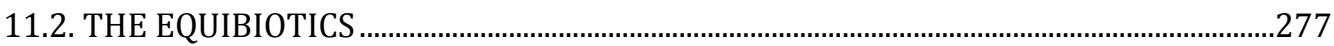

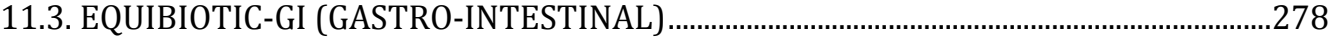

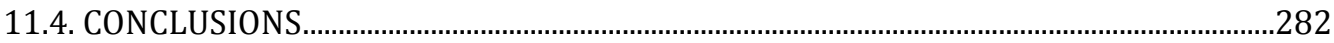

ACKNOWLEDGMENTS....................................................................................................................283

REFERENCES ....................................................................................................................................28 


\subsection{THE HUMAN MICROBIOME}

During the last decades of the twentieth century, the evolution of knowledge on microbial genetics has revealed the existence of a permanent and indispensable functional relationship between microbes of the natural environment and cells of certain tissues of the human body. The first discoveries made about microbes normally present in some tissues of the healthy human being, revealed the role played by what were then called 'intestinal flora' on the digestive function of the large intestine. Subsequent studies led to the discovery and understanding of the existence of a large set of families of microorganisms whose presence was indispensable for the proper functioning of the digestive tract. Later, the confirmed presence of millions of different non-pathogenic microorganisms in the skin, the respiratory and genitourinary tracts came to be, in classic microbiology, interpreted as 'symbiotic' and benefiting the healthy 'host'.

Today the understanding of such important microbe/cell interactions has evolved. The studies and techniques used in the discovery of the human genome applied to the genetic content of the microbial collection permanently lodged in cell tissues of all animals now is considered the microbiome.

According to its definition, the human microbiome is the genetic aggregate of microorganisms that reside on or within any of a number of human tissues and biofluids, including the skin, mammary glands, placenta, seminal fluid, uterus, ovarian follicles, lungs, saliva, oral mucosa, conjunctiva, biliary and the mucous membrane of respiratory and gastrointestinal tracts. They include thousands of bacteria, archaea, fungi and viruses and differ in each person. The term 'human microbiome' refers specifically to the collective genomes of resident microorganisms considered a counterpart to the human genome. The study of the 'communication' of these two great genetic systems results in the key to understand the functioning of the human body [1-3].

Consequently, in this field of research, an ecosystem is defined as the functional unit composed of the local set of microbes (microbiota) and the set of human cells (local tissue) that share a habitat (specific physic-chemical local conditions) that guarantee their interaction. For example, in the ecosystem of the digestive tract, microorganisms find the components they require for their survival and proliferation on the surface of the mucosa, giving rise to the formation of specific microbiota (buccal, oesophageal, gastric, pyloric, duodenal, colonic, rectal, etc.). By finding the right local conditions, the different microbial colonies reproduce and synthesize bioactive compounds (messenger molecules that monitor processes, activate immunological responses, trigger genetic functions, etc.) which, once incorporated into the bloodstream by cells, act remotely on other organs of the human body [4-6]. 
In all these ecosystems, colonies of microbes vary in density (quantity) and diversity (types of families and genera). Depending on the local physicochemical conditions, a state of biological balance or "equilibrium" with the human cells develops at the site. The newest interpretations of this extraordinary phenomenon have allowed us to extend the old concept of homeostasis or 'physiological self-regulation' of the human body. Homeostasis is now considered the result of genetic functional interaction between both the human microbiome and genome. This scope modifies the medical interpretation of the binomial health-disease paradigm [7].

Thus, the origin of infectious or pathogenic microbial colonization processes has to be considered as a rupture of the equilibrium of the microbiota/tissue relationship, a condition called dysbiosis.

In accordance with this scope, the strategy to treat common infectious diseases is now different from that practiced by medicine for most of the $20^{\text {th }}$ century. The previous medical paradigm of maintaining the human body in almost aseptic conditions through the systematic annihilation of pathogenic microbes with potent antibiotics and antiseptics of all kinds has often been counterproductive. The worldwide re-emergence of infectious diseases previously controlled by antibiotics now rendered ineffective is due to the formation of "resistant" microbial strains to such drugs and the emergence of genetic variants of previously non-existent pathogens. In addition, environmental air and water pollutions, chemical composition of modified animal and vegetable foods, among many other factors, have contributed to the origin of multiple functional alterations of the human microbiome [8].

Some of these microbiome alterations are manifested as metabolic disorders (diabetes, obesity, malnutrition) or as central nervous system dysfunctions (insomnia, Alzheimer's disease, infantile dementia), or as altered autonomic manifestations (respiratory and cardiac dysrhythmias, fibrosis, asthma) or by disruptions in the immune system (immuno-deficiencies, allergies), etc. [9-11].

Dysbiosis of the digestive tract microbiota will give rise to many conditions now considered part of the same process. In the mouth, alteration of the local microbiota will produce dental caries and periodontitis; in the stomach, reflux, gastritis, stomach ulcers, dyspepsia; in the intestine, conditions such as irritable bowel syndrome, chronic diarrhoea or constipation, malabsorption, diverticulitis, etc. [12-14].

In the search for solutions to intestinal dysbiosis, the so-called prebiotics (specific nutrients for microbes) have gained great importance as useful functional foods that benefit the organism by stimulating the growth and activity of one or more strains of 'benign' bacteria present in the intestine. Similarly, the probiotics are foods added with living specific microorganisms that remain active in the intestine and have important effects on the constitution of the appropriate microbiota of the digestive tract $[15,16]$. 


\subsection{THE EQUIBIOTICS}

In the last two decades, the renaissance of phytotherapy research all around the world has boosted the scientific study of medicinal plants in many countries. Medicinal plants selected from the so-called 'traditional herbal medicines' used historically by different medical cultures are now scientifically studied in the search for alternative products for the treatment of common infections. The existence of plant remedies with attributed 'antimicrobial' properties represents a huge chapter of herbal medicine in many places [1720].

In Mexico, researchers from our centre have worked in this direction developing phytodrugs made from medicinal plant extracts popularly used to attend to common infectious ailments. The results of this research allowed the designing of a type of plant extract mixture, characterized as 'equibiotics' acting on the maintenance or restoration of the microbiome balance.

According to its definition, equibiotics act simultaneously in two modalities: with auferobiotic and alerebiotic effects. The first refers to the ability of some plant extracts to avoid the proliferation of certain pathogenic microbes and, the second, their complementary capacity to restore conditions that favour the normal balance of a local microbiota [21-23].

The auferobiotic effect is a concept related to several mechanisms of growth inhibition of pathogenic microbes induced by certain bioactive plant compounds. According to the literature, several medicinal plant extracts, considered as 'antimicrobial' agents, possess a capacity not necessarily based on a destructive effect on the pathogen strain. For example, they may inhibit the proliferation of pathogens by a bacteriostatic effect, or paralyzing and impeding the adhesion of certain bacteria to the mucous membranes, or stimulating the production of specific bacteriocins in other 'positive' strains of the ecosystem, etc.

On the other hand, the alerebiotic effect observed during the use of equibiotics is manifested by favouring reconstruction of local tissues, balancing oxidative processes, reducing inflammation, reinforcing local immune responses, or interacting with bacterial genes and inducing proliferation of 'beneficial' strains $[24,25]$.

During the last years, we have been performing studies for the design and use of these kinds of plant extracts. As an example, we describe the characteristics of an equibiotic plant product proposed to balance the microbiota in the digestive tract. 


\subsection{EQUIBIOTIC-GI (GASTRO-INTESTINAL)}

The herbal medicine named Equibiotic-GI, developed for the treatment of chronic dyspepsia, whose more common symptoms are stomach distension and pain, gastritis, nausea, abdominal sensation of fullness, flatulence and chronic colitis with alternative episodes of diarrhoea or constipation [26].

Equibiotic-GI contains two extracts. One is obtained from the leaves of Psidium guajava L. (Myrtaceae) and the second, from the roots of Coptis chinensis Franch. (Racunculaceae). Both plants are used in herbal medicines in several countries of the Americas and Asia, with pharmacological properties widely recognized as anti-microbial, anti-diarrheal, anti-oxidant, spasmolytic, digestive, anti-diabetic and sedative (Figures 1 and 2).

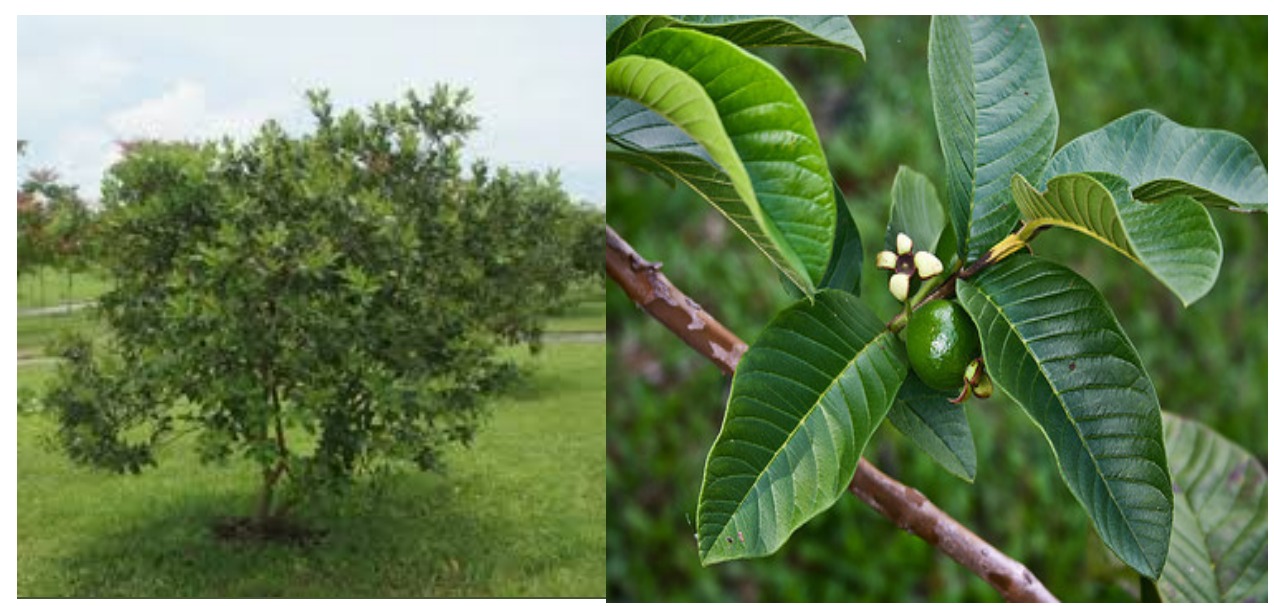

Figure 1. Psidium guajava L. (Fam. Myrtaceae) popularly named Guava; the leaves are used in Mexican traditional medicine in the concoction of tisanes for the treatment of gastrointestinal disorders such as diarrhoea, chronic colitis, gastritis, etc.

Psidium guajava is a species originally from Mexico and Central America and its leaf extracts have been extensively reported in modern scientific literature due to its content of derivatives from flavonoids quercetin and morin, considered bioactive [27]. 


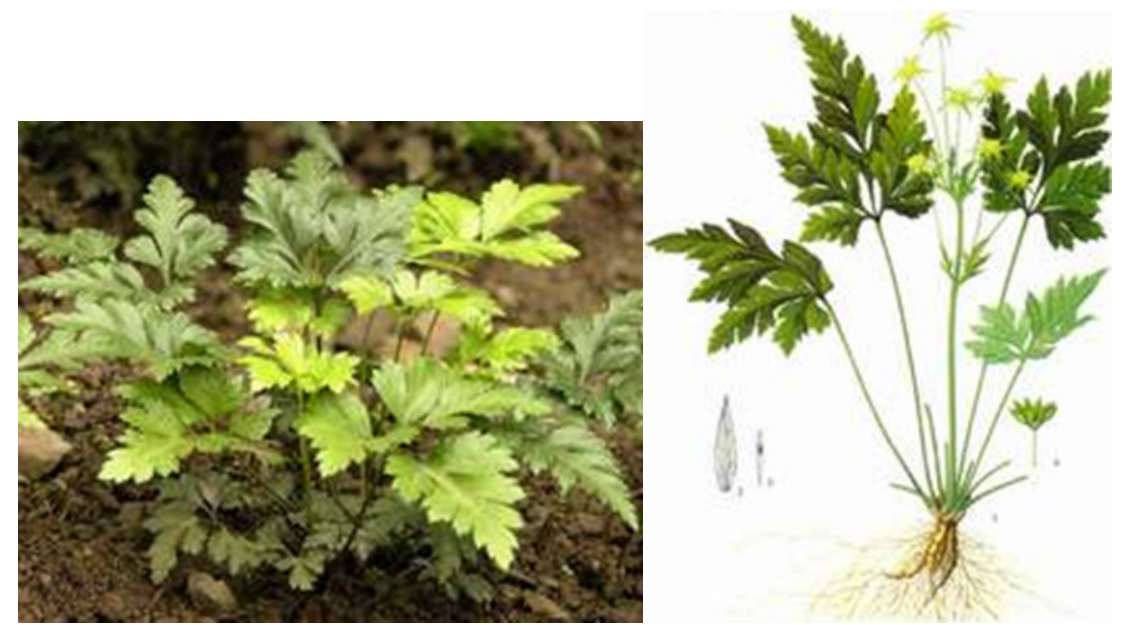

Figure 2. Coptis chinensis Franch. (Fam. Racunculaceae) popularly named Huang Lian. According to traditional Chinese medicine, the root is a pungent, very bitter, cooling herb that controls bacterial and viral infections, relaxes spasms, lowers fevers and stimulates the circulation.

Coptis chinensis root extract is also widely used in Asia for the treatment of gastro intestinal infections, chronic bowel syndrome and diverse digestive ailments, and its bioactivity related to the content in alkaloidal berberine derivatives [28].

Equibiotic-GI is a phytodrug composed of a dry extract of Statum coptis chinensis, standardized in its content of isoquinoline alkaloids, quantified as berberine $(\geq 6.0 \%)$ and a hydro-glycolic extract of Folia psidii guajavae, standardized in its content of flavonic glycosides, quantified as quercetin ( $\geq 0.1 \%$ ). The mixture is pharmaceutically formulated as a suspension for oral administration, for a ten day treatment to alleviate chronic dyspepsia.

Due to the fact that Helicobacter pylori invasion is generally associated with chronic gastritis and, in general, to dyspepsia syndrome, initially the studies that allowed evaluation of the properties of this combination of extracts, were performed with in vitro cultures of several clinically obtained strains of the bacterium $H$. pylori, resistant to conventional antibiotics.

Both extracts inhibited the proliferation of $H$. pylori antibiotic-resistant strains. Minimum inhibitory concentration (MIC) obtained with the mixture of extracts to produce $100 \%$ inhibition of the growth of the bacteria cultures, showed a remarkable synergistic ability to reduce the MIC value by 500 and 1000 times, compared with the test of both extracts separately. The following studies determined the property of the mixture to prevent the adhesion (local and diffuse) of the Helicobacter to gastric epithelial cells (AGS) cultivated in vitro [29]. 
To these multiple in vitro bioactive effects of the plant extract mixture on $H$. pylori, followed conventional preclinical toxicological studies of the pharmaceutical form developed as Equibiotic-GI for oral human administration [30].

Once the non-toxic effects of the product were established, the clinical trial consisted of a Phase III study in a group of volunteers $(n=30)$ with chronic functional dyspesia. The study included the DNA analysis of the intestinal microbiota of the patients before and after administering the Equibiotic-GI. The treatment was given to the participants as home treatment $(20 \mathrm{~mL}$ of suspension, once daily, v.o., during 15 days) maintaining the habitual diet and life style.

At the beginning of the study, a medical interview assessed the clinical history and determined signs and symptoms of functional dyspepsia in every participant. A faecal sample was collected before starting the treatment. In a second session after treatment, a medical interview was performed to determine clinical changes observed and the corresponding faecal sample obtained. Samples from each participant, before and after the study, were processed using conventional methods established to obtain DNA of the intestinal microbiota, determining two levels of genetic identification, by microbial families and by genera [31].

Results obtained showed improvement of all dyspepsia symptoms: drastic disappearance of abdominal pain, reduction of intestinal inflammation and flatulence, marked reduction of episodes of gastritis, disappearance of nausea, increase of appetite and improvement of quality of sleep and mood.

The micro-genomic studies showed a significant increase in the Bacteriodetes families and a moderate reduction in the Firmicutes families, detected in all cases after treatment. In subsequent genera and species analysis, the intestinal microbiota post-treatment changes were qualified as proliferation of positive microbial strains, together with a notable increase in diversity (Figures 3 and 4).

The increase of 'positive genera' was interpreted as manifestations of recovery of the microbiotic normal balance in each individual and, in all cases, included a greater diversity of strains and a significant reduction of groups considered 'potentially pathogenic'. Finally, a predominance of microbial species linked to the reduction of intestinal inflammation and to positive immuno-protective responses against possible pathogens were confirmed in all cases. 


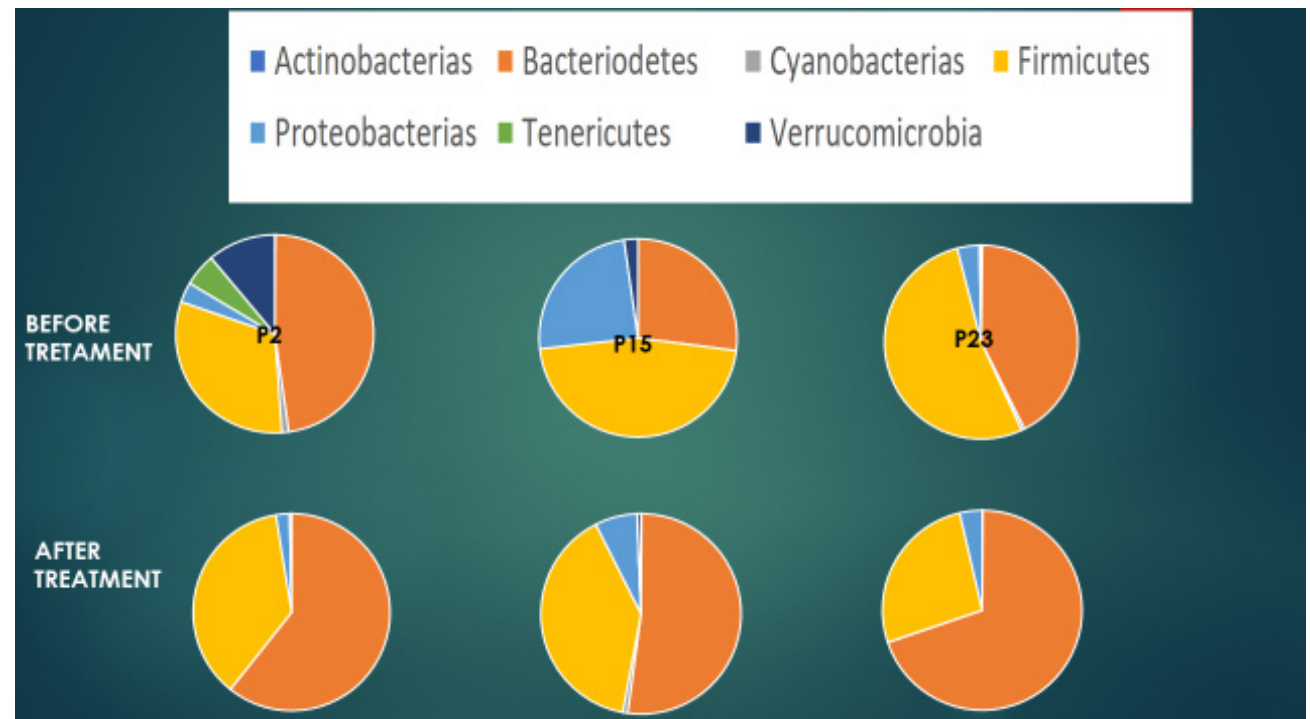

Figure 3. Intestinal microbiograms of three patients (P2, P15, P23) with gastrointestinal dysbiosis, before and after treatment with Equibiotic-GI. Note the increment of Bacteriodetes genera in all cases.

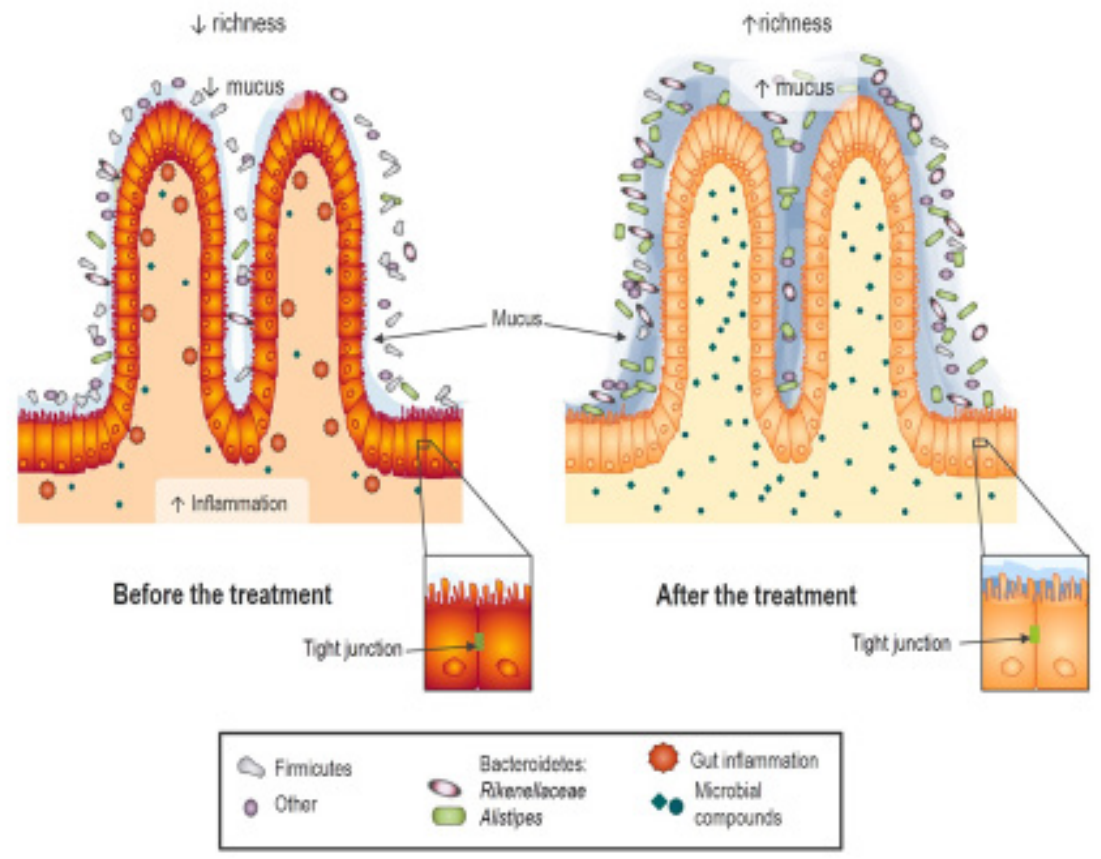

Figure 4. Drawing that illustrates the mucosa of the intestine before and after administration of Equibiotic-GI 


\subsection{CONCLUSIONS}

The extract obtained from Psidium is particularly rich in flavonoids; the most abundant includes quercetin, morin, kamferol, rutin and guajaverin. All of these compounds have recognized anti-microbial activity against very diverse bacterial strains that cause important infections. According to our observations, the nature of this anti-microbial activity is predominantly bacteriostatic, affecting adhesion and not necessarily destroying the strain $[32,33]$. Nevertheless, flavonoids involved in anti-microbial actions may use the following mechanisms:

Inhibition of membrane function affecting its permeability and causing the disruption of its barrier function. This produces the interruption of the normal flow of nutrients, while inducing the loss of essential components for the development and proliferation of the microorganism. With this effect, the motility, chemotaxis, adhesion and invasive capacity of the bacteria should be altered.

Inhibition of the synthesis of nucleic acids (DNA and RNA) through the intercalation of hydrogen bonds in the structure of the nitrogenous bases that form them, and through inhibition of enzymes involved in the synthesis processes of the DNA and RNA of microorganisms.

Inhibition of energy metabolism, acting on the respiratory chain and bacterial electronic transport, affecting the synthesis processes of macromolecules essential for the development of the bacteria. All of these processes can occur simultaneously, depending on the dose and combination of flavonoids used.

Additionally, we add the fact that according to the scientific literature, other plant substrates with recognized anti-microbial properties have been reported and contain alkaloid-type active ingredients, particularly those rich in hydrophobic cations, which attack the membrane itself and the bacterial DNA.

Such is the case of the extracts of plants of the genus Coptis because they contain isoquinolines and many other abundant compounds structurally related to berberine, a hydrophobic cation that easily crosses the microbial membrane and that accumulates inside the bacterium, taking advantage of the alterations it causes in its potential trans-membrane. This property of accumulation of the berberine derivatives inside bacteria is more than 1000 times higher than that of other molecules of plant origin, which makes them essential assets in the sought after anti-microbial effect.

Coptis extract also contains 5'-methoxyhydnocarpine (5'-MHC), a powerful inhibitor of bacteria efflux pumps, which potentiates the antimicrobial action of berberine at very low concentrations. It is interesting to mention that the 5 'MHC has a high chemical structural analogy with the flavonoid quercetin and that its inhibitory capacity for microbial growth related to the glycoproteins that operate the microbial efflux pumps (MDRs). 
In addition, everything seems to indicate that the flavonoid and alkaloidal effects are also related to their ability to interfere with efflux pumps and the mechanism of "quorum sensing" (QS) of undesirable bacteria in the ecosystem, a complex mechanism of action yet to be tested experimentally [34-38].

Based on the above, data indicates that the extracts of Psidium guajava and of Coptis chinensis, mixed in a range of synergistic proportions, owe their peculiar auferobiotic effect mainly to the combination of the quercetin and berberine derivatives $[39,40]$.

On the other hand, the alerebiotic effect of the phytodrug Equibiotic-GI, was verified by the positive changes provoked in the composition of the gut microbiota of patients with functional dyspepsia. The observation also corroborates the relationship that exists between symptomatic relief and therapeutic benefits with the configuration of the microbiota.

In this sense, we demonstrated the so-called equibiotic effect by corroborating that a state of "normal" or "in equilibrium" intestinal microbiota can be induced. However, the complex mechanisms that are involved in this phenomenon need still to be deeply investigated.

\section{ACKNOWLEDGMENTS}

The clinical studies for the development and assessment of the Equibiotic-GI were carried out in the Infectious Diseases Research Unit of the Mexican Institute of Social Security (IMSS), in the Preclinical Research Unit (UNIPREC) of the National Autonomous University of Mexico (UNAM) and in the National Institute of Genomic Medicine (INMEGEN).

\section{REFERENCES}

1. R.R. Dietert, The Human Superorganism: how the microbiome is revolutionizing the pursuit of a healthy life. Penguin Random House, Dutton, New York, 2016. ISBN 9781101983904.

2. NIH Human Microbiome Working Group. "The NIH Human Microbiome Project" , Genome Res. 19(12) (2009) 2317-2323.

3. D.A. Relman, Nature 486 (2012) 194-195.

4. J. Li, X. Cai, H. Zhong, et al., Nat. Biotechnol. 32 (2014) 834-841.

5. S. Greenblum, R. Carr, E. Borenstaein, Cell 160(4) (2015) 583-594.

6. L. Liu, X. Chen, G. Skogerbø, et al., Genomics 100(5) (2012) 265-270.

7. A. Hoffman, C.A. Zimmermann, D. Spengler, Front. Behav. Neurosci. 13(9) (2015) 120.

8. M.J. Blaser. Missing microbes: how the overuse of antibiotics is fueling our modern plagues. Henry Holt \& Co., New York, NY.2015.

9. R.R. Dietert, J. Dietert, Entropy 14(11) (2012) 2036-2065. 
10. B. Owens, Nature News 495(7441) (2013) 300-303.

11. L.S. Weyrich, S. Dixit, A.G. Farrer, A.J. Cooper, Australas J. Dermatol. 56(4) (2015) 268-274.

12. A.T. Stefka, T. Feehley, P. Tripathi, et al., Proc. Natl. Acad. Sci. U S A 111(36) (2014) 13145-13150.

13. T. Patel, P. Bhattacharya, S. Das, Gastrointest. Cancer 47(3) (2016) 232-238.

14. G. Cammarota, G. Ianiro, R. Cianci, S. Bibbò, et al., Pharmacol. Ther. 149 (2015) 191-212.

15. M. Roberfroid, G.R. Gibson, L. Hoyles, et al., J. Nutr. 104(2) (2010) S1-S63.

16. C.J. Walsh, C.M. Guinan, P.W. O'Toole, P.D. Cotter, FEBS Lett. 588 (22) (2014) 4120-4130.

17. S. Qin, J. Li, C. Hua-Hong, et al., Appl. Environ. Microbiol. 75(19) (2009) 61766186.

18. J.P. Piwowarski, S. Granica, M. Zwierzyńska, et al., Ethnopharmacol. 155(1) (2014) 801-809.

19. L. Chen, W.C.S. Tai, M.S. Brar, et al., PLoS One 10(5) (2015) e0126807.

20. F. Chen, Q. Wen, J. Jiang, et al., J. Ethnopharmacol. 179 (2016) 253-264.

21. G. Ianiro, S. Bibbo, A. Gasbarrini, et al., Curr. Drug Targets 15(8) (2014) 762770.

22. X. Lozoya, M. Gascón-Muro, J. Agüero, et al., Rev. Fitoterapia 12(2) (2012) 145148.

23. D.A. Spratt, M. Daglia, A. Papetti, et al., J. Biomed. Biotechnol. 2012 (2012) 510198.

24. X. Lozoya, M. Gascon-Muro, J. Aguero, E. Rivera, Equibiotic compositions for the treatment of periodontal disease and halitosis in animals. Patent $\mathrm{Mx} / \mathrm{E} / 2012 / 014315$.

25. X. Lozoya, M. Gascon-Muro, J. Aguero, E. Rivera, Composiciones equibioticas de extractos de plantas útiles para el tratamiento de la infección gástrica por Helicobacter pylori. Patent MX(a/2015/005527.

26. G. Chiaroni, M. Pesce, A. Fantin, et al., Unit. Eur. Gastroenterol. J. 6(1) (2018) 512.

27. X. Lozoya, E. Rivera, et al., Psidium guajava L. (myrtaceae) in: Tortoriello García J and Osuna Torres L. (editors), "Investigacion Científica de la Herbolaria Mexicana” Ed. IMSS, Mexico, 2016; Chapter V, pp. 63-88.

28. Coptis chinensis, WHO Selected Medicinal Plants Monographs 1(12) (1999) 15-41.

29. X. Lozoya, J. Aguero, M. Gascón-Muro, et al., Revista de Fitoterapia 15(2) (2015) 101-113.

30. I. Gracia Mora, L. Macias Rosales, et al., Evaluacion de la seguridad (Toxicidad aguda y Genotoxicidad) de la combinación de Psidium guajava L y Coptis chinensis Franch. Unidad de Investigacion Preclinica UNIPREC, Universidad Nacional Autonoma de Mexico UNAM 2016; IF: 1-25.

31. B. Palacios-Gonzalez, et al., Equibiotic-GI balances intestinal microbiota in subjects with functional dyspepsia. 2017 (in press).

32. F.I. Brighenti, S.B.I. Luppens, -A.C.B. Delbem, et al., Caries Res. 42(2) (2008) 148-154.

33. C. Ankolekar, T. Terry, K.C. Johnson, et al., J. Med. Food 14(11) (2011) 13211329.

34. G. Spegler, Molecules 22 (2017) 468. 
35. T. Ohene-Agyei, Microbiol. Open 3(6) (2014) 885.

36. C. Anh Kim Ta, Molecules 21 (2016) 29.

37. H. Asfour, J. Microscop. Ultrastruct. 2017 (in press).

38. H. Vasavi, J. Microbiol. Immunol. Infect. 49 (2016) 8.

39. F.R. Stermitz, P. Lorenz, J.N. Tawara, et al., Proc. Natl. Acad. Sci. USA 97(4) (2000) 1433-1437.

40. A.R. Ball, G. Casadei, S. Samosorn, et al., ACS Chem Biol 1(9) (2006) 594-600.

(C)2018 by the authors; licensee IAPC, Zagreb, Croatia. This chapter is an open-access publication distributed under the terms and conditions of the Creative Commons Attribution license (http://creativecommons.org/licenses/by/3.0/) (cc) EY 
